\title{
Perception of degraded vocalizations by budgerigars (Melopsittacus undulatus)
}

\author{
THOMAS J. PARK and ROBERT J. DOOLING \\ University of Maryland, College Park, Maryland
}

\begin{abstract}
Three budgerigars were trained with operant techniques to discriminate examples of speciesspecific contact calls. Once the birds learned the discrimination among a large set of calls, they were tested with the calls altered in various ways. The birds' performance on altered calls was compared with performance on normal calls. Results show that the birds could tolerate large spectral and temporal alterations in the calls with little or no decrement in performance. A comparison of the birds' performance across conditions shows clear evidence of a redundancy in informa. tion contained in the calls. The birds were equally adept at discriminating among the calls when given only high-frequency information and when given only low-frequency information. The birds also performed just as well when presented with only the short beginning portions of the calls as they did when presented with somewhat longer portions near the end of the calls. This ability of budgerigars to discriminate among species-specific vocal signals that are highly distorted is reminiscent of the ability of humans to discriminate and recognize highly distorted speech sounds.
\end{abstract}

The study of bird vocalizations over the past few decades has provided some of the most sophisticated examples known of complex learning among animals (Kroodsma \& Miller, 1982; Marler, 1970). Unfortunately, the focus of much of this research has been restricted to the assessment of vocal output, while the measurement of the perceptual processes involved has been somewhat neglected. It has generally proven easier to record and quantify the vocal behavior of small birds than to design experiments to directly test auditory processing. The budgerigar, or parakeet, represents somewhat of an exception in that it has proven to be an extremely tractable species for studying auditory perception in the laboratory, using operant techniques (Dooling \& Saunders, 1975; Dooling \& Searcy, 1985; Park \& Dooling, 1985; Park, Okanoya, \& Dooling, 1985).

Recent experiments on hearing in the budgerigar have demonstrated that this species has unusually good hearing in the spectral region of 2-4 kHz (Dooling \& Saunders, 1975; Dooling \& Searcy, 1985; Saunders, Rintelmann, \& Bock, 1979). Furthermore, the budgerigar can learn and remember for long periods of time a discrimination among a large set of vocalizations called contact calls (Dooling, 1986; Park \& Dooling, 1985). The exact acoustic cues that budgerigars rely on in discriminating among contact calls is unknown, but the spectral region of 2-4 $\mathrm{kHz}$ seems to be particularly important (Park \& Dooling, 1985).

Field studies have shown that: (1) almost any acoustic cue can be used for species recognition by voice in birds

These experiments were supported by Public Health Service Grant NS-19006 and Research Career Development Award HD-00512 to R. Dooling. We thank Brian Dooling for care of the birds and S. Brown, S. Hulse, K. Okanoya, and J. Sinnott for comments on an earlier draft. Requests for reprints should be sent to R. Dooling, Department of Psychology, University of Maryland, College Park, MD 20742. (i.e., note duration, internote interval, pitch, presence of harmonics), and (2) there are tremendous species differences in the acoustic cues that are used (Becker, 1982; Falls, 1982; Peters, Searcy, \& Marler, 1980). The purpose of the present experiments was to explore the basis of contact call perception in budgerigars. We were specifically interested in determining whether budgerigars relied on some critical subset of acoustic features in discriminating among contact calls or whether they were capable of using any acoustic difference between calls in making a discrimination. The modification of complex acoustic signals, such as the budgerigar contact call, can be a difficult and involved process. Since there were no data available on which acoustic features of the contact call might be important for the budgerigar, we chose to start with the two simplest manipulations possible in degrading these natural vocal signals.

\section{METHODS}

\section{Subjects}

Three budgerigars (Melopsittacus undulatus) were used as subjects in the present experiments. These birds, which had been obtained from commercial dealers, had had previous experience in an operant testing situation using contact calls as discriminative stimuli (Park \& Dooling, 1985). Yellow millet was used as a reinforcer during experimental sessions, and standard mixed parakeet seed was available during free-feeding times.

\section{Apparatus}

The apparatus used for training and testing the birds has been previously described (Park \& Dooling, 1985; Park et al., 1985). Briefly, a standard operant conditioning apparatus normally used for pigeons was modified by the addition of a response panel consisting of two response keys (observation key and report key) made of microswitches and LEDS. This panel was mounted directly above the opening of a food hopper, and the bird could easily trip the microswitches by striking the LEDS with its beak. Experimental events were controlled by a DEC PDP-11/23 microcomputer oper- 
ating a Dynaport Serial Interface (Coulbourn Instruments). Vocalizations were stored in digital form on hard disk and played out during a trial at a sampling rate of $20 \mathrm{kHz}$ through a digital-to-analog converter. Calls were presented at a peak sound pressure level of $85 \mathrm{~dB} \pm 2 \mathrm{~dB}$ SPL measured at the position of the bird's head.

\section{General Procedure}

The general procedures for training and testing the birds have also been described in detail (Park \& Dooling, 1985; Park et al. 1985). The birds were trained on a go/no-go operant procedure to discriminate one set of seven contact calls (go stimuli) from a second set of seven contact calls (no-go stimuli). Each call was recorded from a different individual and, for convenience, the calls were grouped by sex. Previous experiments had established that there were no systematic acoustic differences between the contact calls of male and female budgerigars (Dooling, 1986). Sonograms and time waveforms of these calls are shown in Figure 1.

A trial began with a response on the observation key. Four repetitions of a contact call were presented with an interstimulus interval of $250 \mathrm{msec}$. If the call was a go stimulus, a response on the report key within $4.0 \mathrm{sec}$ resulted in a 2 -sec access to grain. Failure to respond within $4.0 \mathrm{sec}$ resulted in a 10 -sec time-out period, during which the lights in the chamber were extinguished. If the contact call was a no-go stimulus and $4.0 \mathrm{sec}$ elapsed without a response on the report key, another trial was initiated. An incorrect response to a no-go stimulus (a response on the report key during the 4-sec response interval) also resulted in a 10 -sec time-out period during which the chamber lights were extinguished.

The birds were trained in daily sessions consisting of 70 trials. Each session consisted of an equal number of go and no-go trials selected in random order with the constraint that the same trial type not occur more than twice in a row. Although this makes the trial type following two similar trials predictable, analysis of the data showed that the birds were not using this strategy. Each of the 14 contact calls was presented five times in each session. Each bird completed two sessions per day-one in the morning and one in the afternoon. There was no difference in performance between morning and afternoon sessions. After the birds reached the $85 \%$ correct criterion level of responding, testing began with altered calls.

\section{EXPERIMENT 1: \\ Perception of Filtered Contact Calls}

Experiment 1 was designed to test whether budgerigars relied on specific spectral cues in maintaining the learned discrimination among species-specific contact calls. By filtering the calls at various frequencies, it was possible to attenuate selected spectral information. A General Radio Universal Filter (Model 1982) was inserted in the audiometric circuit, which filtered out high- and lowfrequency energy in the call at the rate of $48 \mathrm{~dB} /$ octave. The birds were then tested on either high- or low-passfiltered calls.

\section{Procedure}

In this experiment, as in training, the birds were tested in two sessions a day. In the morning, the birds were tested on the set of 14 unfiltered contact calls. In the afternoon, the birds were tested on the same set of calls but with the filter set for either low or high pass at a frequency of $2,3,4$, or $5 \mathrm{kHz}$. The order of testing of the different condi-

\section{BUDGERIGAR CONTACT CALLS}

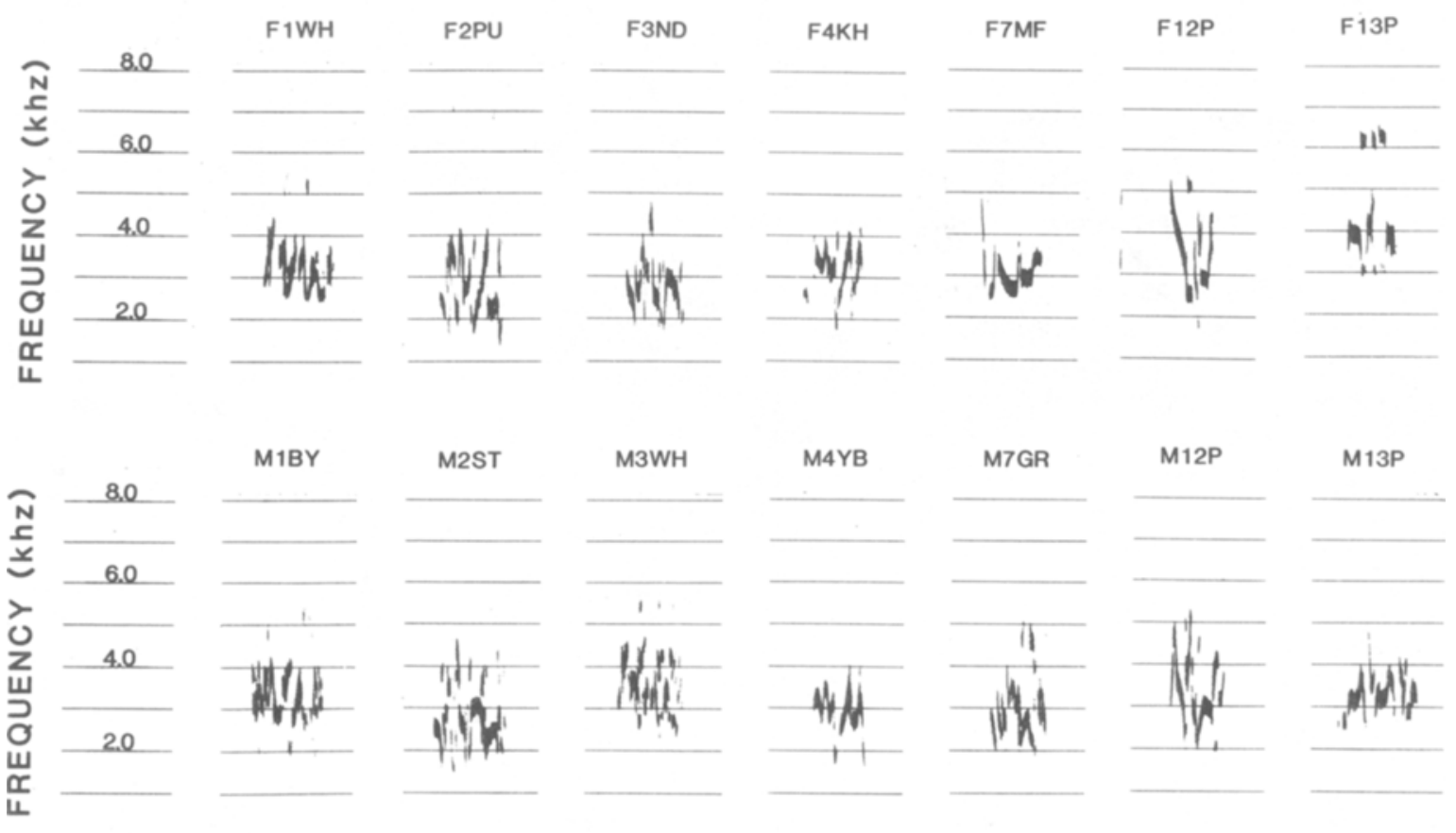

Figure 1. Sonograms of the 14 budgerigar contact calls used as test stimuli. Frequency is on the ordinate in kilohertz. The time marker is 200 msec. 
tions was randomized, and a different random order was used for each bird. The effect on performance of a particular filtering condition was taken as the difference between the first (unfiltered calls) and second sessions each day. As in the training sessions, all sessions in this experiment consisted of a total of 70 trials, or 5 trials for each contact call.

As a check on whether the effect of filtering the calls was peculiar to species-specific vocalizations, two of the birds were also trained and tested on a set of 14 canary calls. Sonograms of these calls are shown in Figure 2. Previous work had shown that budgerigars could learn to discriminate among canary calls as efficiently as they could learn to discriminate among species-specific calls (Park \& Dooling, 1985).

\section{Results}

The results of Experiment 1 are shown in Figure 3. In a comparison of the low-pass filtering conditions and baseline performance, the birds showed no deficit with lowpass filtering settings of $5 \mathrm{kHz}[t(3)=0.0$, n.s.] and $4 \mathrm{kHz}[t(3)=1.00$, n.s. $]$. However, below $4 \mathrm{kHz}$, performance declined significantly relative to baseline performance as the filter setting was decreased to $2 \mathrm{kHz}$.

Conversely, in the high-pass filtering condition, performance declined as the filter setting was increased from 3 to $5 \mathrm{kHz}$. The effects of high-pass and low-pass filtering of the calls are equivalent (a relatively small deficit in both cases) at about $3 \mathrm{kHz}$ - the approximate center of the frequency band containing most of the energy in the contact calls (see Figure 1). Presenting energy in the spectrum only below $[3.0 \mathrm{kHz}$ low pass; $t(3)=1.03$, n.s.] or only above [ $3.0 \mathrm{kHz}$ high pass; $t(3)=.55$, n.s.] the center of the spectrum did not result in a significant decrement in performance. In each filtering condition, the er- rors for all 3 birds were distributed equally across the 14 contact calls. Thus, filtering did not have a differential effect on particular calls.

The results for the two birds that were also trained and tested on canary calls are shown in Figure 4 . The effect on performance of filtering canary calls is similar to that found for filtering budgerigar calls.

\section{EXPERIMENT 2: \\ Perception of Shortened Contact Calls}

The previous experiment demonstrated that the performance of budgerigars in discriminating among contact calls is just as good when they are given only highfrequency information as when they are given only lowfrequency information. Experiment 2 was designed to test whether budgerigars rely on cues that occur at specific temporal locations within the calls (i.e., beginning, middle, or end) in maintaining a discrimination among the set of contact calls. For this experiment, an electronic switch (Coulbourn Instruments Model S84-04) was inserted in the audiometric circuit. The birds were then tested with selected portions of the calls removed.

\section{Procedure}

As in Experiment 1, the birds were tested in two sessions per day. The first session consisted of a test on the 14 calls unmodified in any way. In the second daily session, the electronic switch was gated in such a way that only the beginning segment of a call (i.e., the first $25 \mathrm{msec}$ ) was presented or the beginning segment of a call

\section{CANARY CONTACT CALLS}

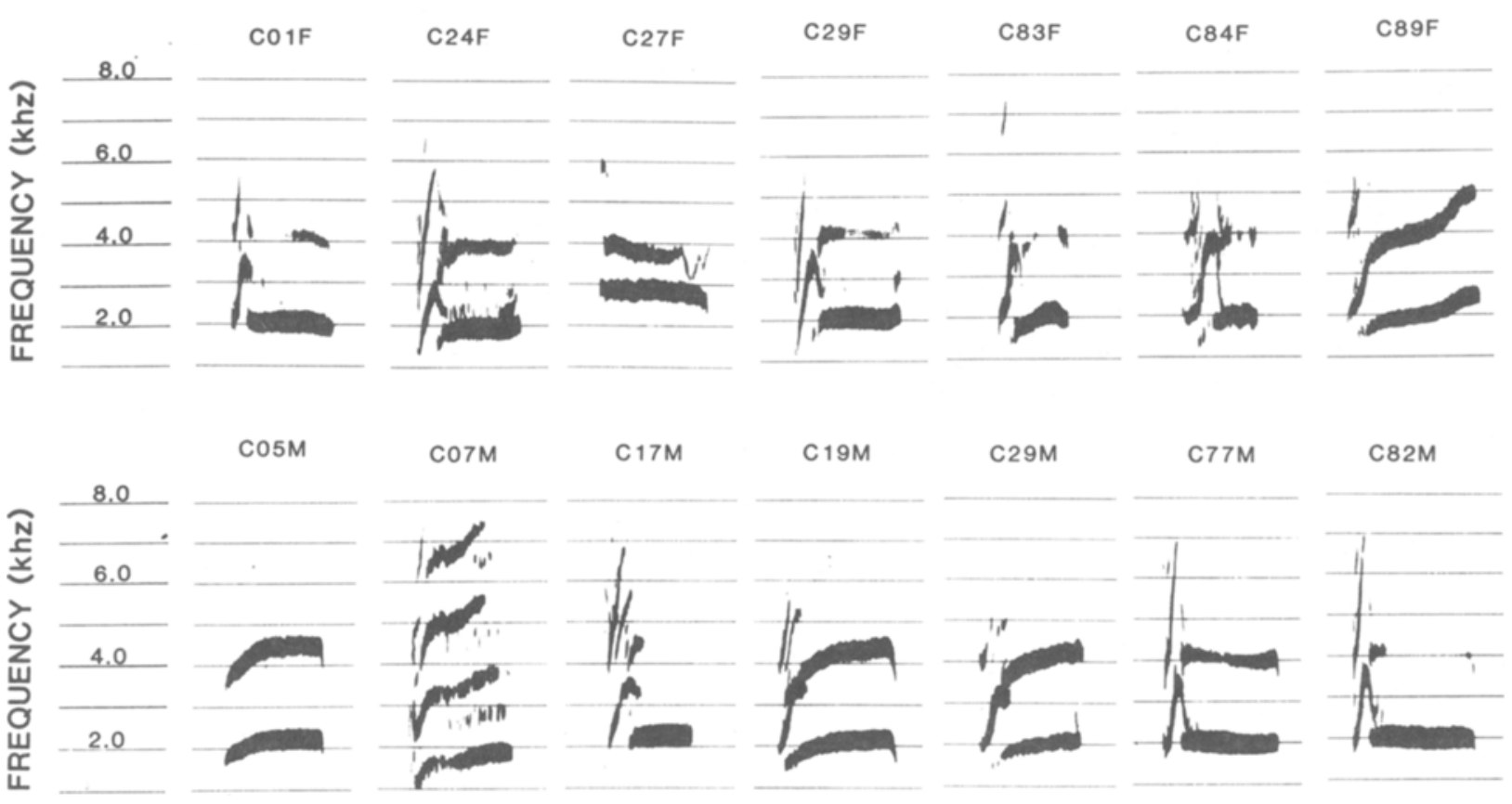

Figure 2. Sonograms of the 14 canary contact calls used as test stimuli. Frequency and time scale are as in Figure 1. 


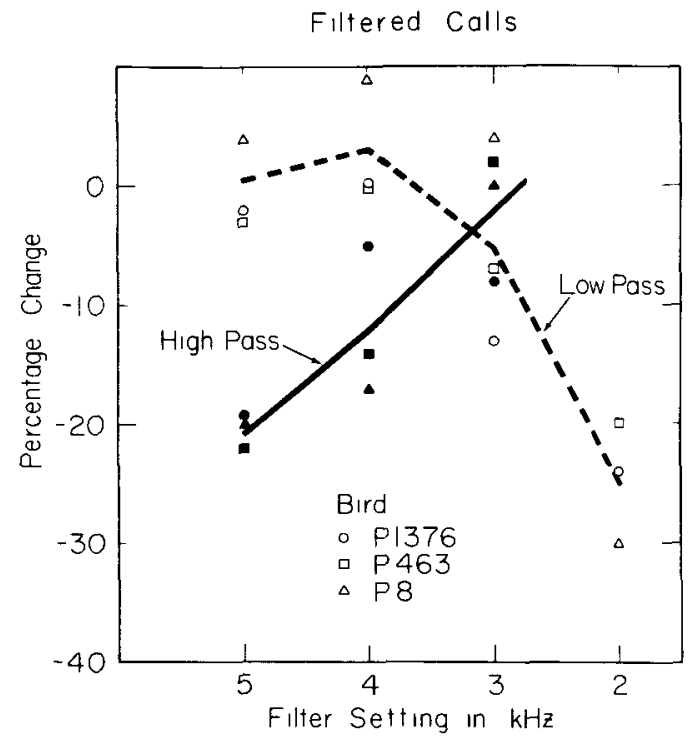

Figure 3. Performance of 3 budgerigars on filtered calls. Performance of the 3 birds on the low-pass-filtered calls is shown by a dashed line, with individual data points shown by open symbols. Performance of the 3 birds on the high-pass-filtered calls is shown by a solid line and solid symbols.

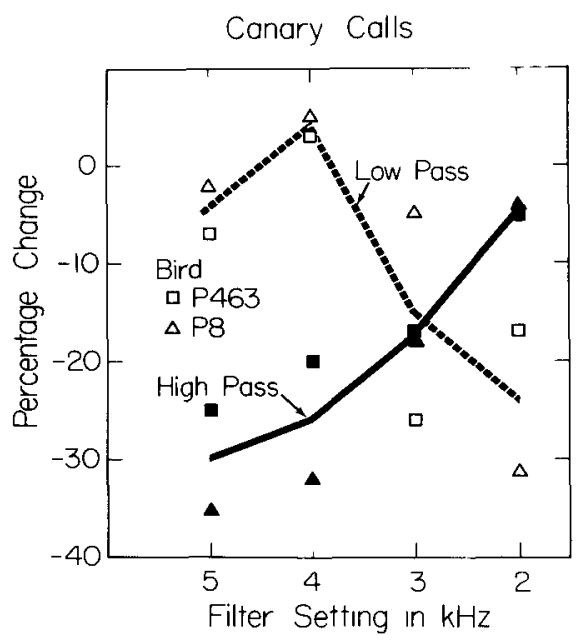

Figure 4. Performance of 2 budgerigars on filtered canary contact calls. Performance on low-pass-filtered calls is shown by a dashed line and open symbols. Performance on high-pass-filtered calls is shown by a solid line and solid symbols.

was omitted. Since the average duration of these calls was about $150 \mathrm{msec}$, omitting the first $75 \mathrm{msec}$ of the call is approximately equivalent to presenting the last $75 \mathrm{msec}$ of the call. In all, there were 10 conditions tested in this experiment. The first $100,75,50$, 25 , or $10 \mathrm{msec}$ of the calls were presented, the first $100,75,50$, or $25 \mathrm{msec}$ of the calls were omitted, or a $75-\mathrm{msec}$ segment in the middle of the calls (beginning $25 \mathrm{msec}$ from call onset) was presented. As in the first experiment, the effect on performance of these acoustic degradations was taken as the difference between performance in the first session (intact calls) and performance in the second.

\section{Results}

The results of Experiment 2 are shown in Figure 5. The birds show a small, but significant, deficit if only the initial $75 \mathrm{msec}[t(3)=5.2, \mathrm{p}<.01]$ to $100 \mathrm{msec}[t(3)=$ $8.66, p<.01]$ of the calls are presented. Interestingly, a similar decrement is observed if everything but the first $25 \mathrm{msec}$ of the call is presented $[t(3)=18.86, p<.001]$. In both cases, the mean difference from baseline is about 9\%.

Performance drops dramatically when less than the first 75 msec of the calls is presented and when more than the first $25 \mathrm{msec}$ of the calls is omitted. There is an approximately monotonic relationship between the decrement in performance and the amount of the initial part of the call omitted. Presenting a 75-msec portion from the middle or end of the call (initial $75 \mathrm{msec}$ omitted) resulted in considerably poorer performance than when only the first $75 \mathrm{msec}$ of the call was presented. Presenting the first $50 \mathrm{msec}$ or all but the first $50 \mathrm{msec}$ of the call resulted in equivalent performance $[t(3)=1.05$, n.s.]. When only the first $10 \mathrm{msec}$ of the calls was presented, performance for all 3 birds fell to a level of chance.

\section{DISCUSSION}

The present investigation demonstrates that there is considerable redundancy of information in the perception of complex contact calls by budgerigars. We conclude that, in discriminating among complex bird vocalizations, budgerigars are not rigidly constrained to acoustic cues available in only a certain spectral or temporal region.

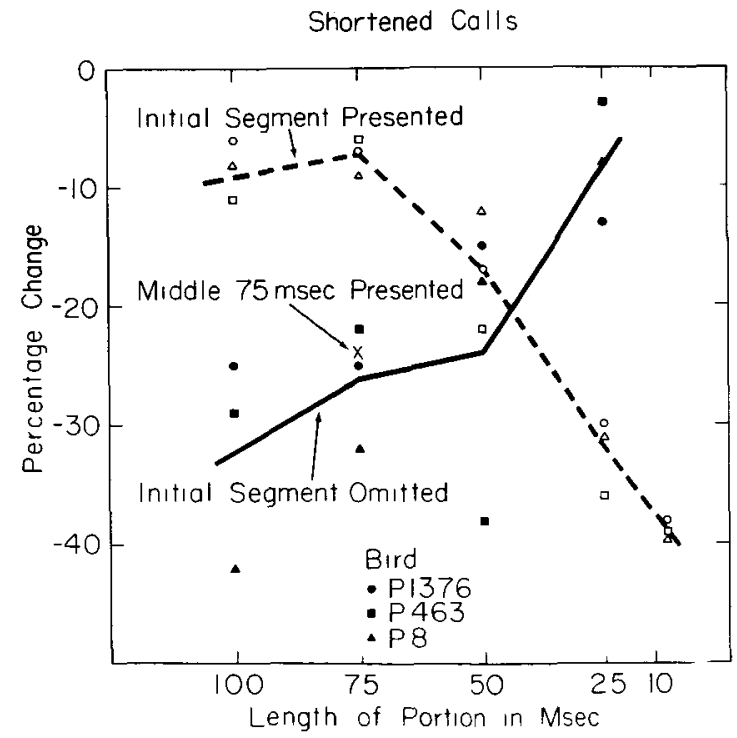

Figure 5. Performance of the 3 budgerigars on shortened calls. Performance when the initial segments of the calls were presented is shown by a dashed line and open symbols. Performance when the initial segments of the calls were omitted is shown by a solid line and closed symbols. The average performance of the 3 birds on a 75 -msec segment taken from the middle of the calls is shown by an $x$. 
The results from the filtering experiment show that budgerigars perform just as well when given only highfrequency information as they do when given only lowfrequency information. The conclusion from presentation of only portions of the calls is that budgerigars rely more on information presented at the beginning of the call than they do on that presented in the middle or at the end of the call. However, even here, there is flexibility in that information in the beginning of the call is not critical. Presenting all but the first $50 \mathrm{msec}$ of the call is equivalent, in terms of performance, to presenting only the first $50 \mathrm{msec}$ of the call. In each case, there is a surprisingly small decrement in discrimination performance.

The flexibility budgerigars demonstrate in their use of spectral cues in discrimination of complex bird calls is reminiscent of that reported for human listening to speech. Humans can recognize speech sounds very well with only the frequencies above or below about $1900 \mathrm{~Hz}$ present in the speech waveform (French \& Steinberg, 1947; Miller, 1981). In other words, there is sufficient redundancy in natural speech for no particular portion of the speech spectrum to be crucial to discrimination. The same appears to be true for budgerigars listening to contact calls.

The bias that budgerigars show for information in the early part of the call is not without precedent-at least among birds. Sinnott (1980) has shown that red-winged blackbirds (Agelaius phoeniceus) and brown-headed cowbirds (Molothrus ater) focus primarily on initial information in discriminating among songs in an operant situation. Hulse and Cynx (in press) have also recently demonstrated that starlings (Sturnis vulgaris), trained on tone patterns varying in pitch, respond preferentially to early rising/falling pitch information in a pitch sequence, to the exclusion of later pitch even when it contradicts early information. Interestingly, these results are in contrast to what is known about the perception of tone patterns by humans. When listening to 10 -tone sequences, untrained human listeners resolve high-frequency components near the end of the patterns better than they do low-frequency components near the beginning of the patterns (Watson \& Kelly, 1981).

There is a wealth of evidence demonstrating that relatively small acoustic changes in vocal signals can have a dramatic effect on vocal recognition. Some of the best known examples come from both field and laboratory studies in birds (Becker, 1982; Falls, 1982; Peters, Searcy, \& Marler, 1980), monkeys (Zoloth et al., 1979), and frogs (Gerhardt, 1986). The results reported here for the budgerigar are not necessarily in contrast to the above findings on the perception of complex species-specific vocalizations by other animals. Consider an example from human speech. Even though humans can tolerate a tremendous amount of distortion in speech, it is also a fact that relatively small acoustic changes in speech can have a profound effect on our percept as. for instance, in the pho- nemic distinction between / da/ and / ta/ (Lisker \& Abramson, 1970; Soli, 1983).

Another point to keep in mind is the methodological difference between the present experiments and other experiments, such as playback studies in the field, that attempt to address the same issues. In the present experiments, the birds may have been well aware that the stimuli had changed when they were degraded. The present procedure speaks only to the issue of whether degradation does or does not disrupt discrimination performance.

The fact that budgerigars can tolerate a tremendous amount of distortion in discriminating among a set of species-specific contact calls should not be too surprising. It is easy to imagine situations in the budgerigars' natural environment in which this capability would prove useful in facilitating communication among conspecifics. The capability for maintaining acoustic communication under one set of adverse conditions does not preclude the possibility that budgerigars might, under other circumstances, rely on relatively minor changes in a specific acoustic feature or combination of features in their vocal signals.

\section{REFERENCES}

BeCkER, P. H. (1982). The coding of species-specific characteristics in bird sounds. In D. Kroodsma \& E. Miller (Eds.), Acoustic communication in birds (Vol. 1; pp. 214-252). New York: Academic Press.

Dooling, R. J. (1986). Perception of vocal signals by budgerigars (Melopsittacus undulatus). Experimental Biology: Environmental \& Sensory Aspects, 45, 195-218

Dooling, R. J., Saunders, J. C. (1975). Hearing in the parakeet (Melopsittacus undulatus): Absolute thresholds, critical ratios, frequency difference limens, and vocalizations. Journal of Comparative \& Physiological Psychology, 88, 1-20

DoOling, R. J., SEARCY, M. H. (1985). Nonsimultaneous auditory masking in the budgerigar (Melopsittacus undulatus). Joumal of Comparative Psychology, 99, 226-230.

Fal.LS, J. B. (1982). Individual recognition by birds. In D. Kroodsma \& E. Miller (Eds.), Acoustic communication in birds (Vol. 1, pp. 237278). New York: Academic Press.

French, N. R. S Steinberg, J. C. (1947). Factors governing the intelligibility of speech sounds. Joumal of the Acoustical Society of America, 19, 90-119.

GERHARDT, C. H. (1986). Sound pattern recognition in treefrogs. Experimental Biology: Environmental \& Sensory Aspects, 45, 167-178.

Hulse, S. H., CYNX, J. (in press). Interval and contour in serial pitch perception by a passerine bird, the European starling (Stumis vulgaris). Journal of Comparative Psychology.

Kroodsma, D., Mrller, E. (1982). Acoustic communication in birds. New York: Academic Press.

Lisker, L., A Aramson, A. S. (1970). The voicing dimension: Some experiments in comparative phonetics. In Proceedings of the 6th International Congress of Phonetic Sciences, Prague, 1967 (pp. 563 567). Prague: Academia.

MARLER, P. (1970). A comparative approach to vocal learning: Song development in white-crowned sparrows. Joumal of Comparative \& Physiological Psychology, 71, 1-25

Milier. G. A. (1981). Language and speech. San Francisco: W, H. Freeman.

PARK. T J. \& Dooling, R. J. (1985). Perception of species-specific contact calls by budgerigars (Melopsittacus undulatus). Journal of Comparative Psvchologv, 99. 391-402. 
Park, T. J., Okanoya, K. \& Dooling, R. (1985). Operant conditioning of small birds for acoustic discrimination. Journal of Ethology (Japan), 3, 5-9.

Peters, S., Searcy, W. A., \& Marler, P. (1980). Species song discrimination in choice experiments with territorial male swamp and song sparrows. Animal Behaviour, 28, 393-404.

Saunders, J. C., RintelmanN, W. F., \& Bock, G. R. (1979). Frequency selectivity in bird and man: A comparison among critical ratios, critical bands, and psychophysical tuning curves. Hearing Research, 1, 303-323.

SinnotT, J. M. (1980). Species-specific coding in birdsong. Journal of the Acoustical Society of America, 68, 862-884.

Solı, S. D. (1983). The role of spectral cues in the discrimination of voice onset time differences. Journal of the Acoustical Society of America, 73, 2150-2165.

WATSON, C. S., \& KELLY, W. J. (1981). The role of stimulus uncertainty in the discrimination of auditory patterns. In D. J. Getty \& J. H. Howard (Eds.), Auditory and visual pattern recognition (pp. 37-59). Cambridge, NJ: Erlbaum.

Zoloth, S. R., Petersen, M. R., Beecher, M. D., Green, S. Marler, P., Moody, D. B., \& Stebbins, W. (1979). Species-specific perceptual processing of vocal sounds by monkeys. Science, 204, 870-873.

(Manuscript received January 13, 1986; revision accepted for publication May 30, 1986.) 